\title{
UAS Icing Physics and Innovative Strategies for UAS Icing Mitigation
}

\author{
Dr. Hui Hu \\ Martin C. Jischke Professor in Aerospace Engineering \\ Director, Aircraft Icing Physics and Anti-/De-icing Technology Laboratory \\ Department of Aerospace Engineering, Iowa State University, USA
}

\section{Extended Abstract}

In-flight icing is widely recognized as a significant hazard to both unmanned and manned aircraft flying in cold climate. In comparison with conventional, large-sized manned aircraft, lightweight Unmanned Aerial Systems (UAS) is more susceptible to inflight icing problems due to the lower cruising altitude with relatively higher liquid water content (LWC) and warmer air temperatures, smaller excess power margin to offset the increased drag caused by icing, lower flying velocity to result in longer exposing to icing conditions, and more damage to important sensors onboard. In this talk, the speaker will introduce the recent progress made in his research laboratory to elucidate underlying physics pertinent to UAS icing phenomena and to explore innovative strategies for UAS icing mitigation. By leveraging a unique Icing Research Tunnel available at Iowa State University (ISU-IRT), comprehensive experimental investigations are conducted to examine the important micro-physical processes pertinent to UAS icing phenomena. A suite of advanced flow diagnostic techniques, which include molecular tagging velocimetry and thermometry (MTV\&T), digital image projection (DIP), and high-speed infrared (IR) imaging thermometry techniques, are developed and applied to achieve quantitative measurements to quantify the impinging dynamics of super-cooled water droplets, transient behavior of the wind-driven surface water runback, unsteady heat transfer and dynamic ice accretion process over the surfaces of UAS wings and rotating propellers. The potentials of various bio-inspired icephobic coatings, including lotus-inspired superhydrophobic coatings and pitcher-plant-inspired Slippery Liquid-Infused Porous Surfaces (SLIPS), for UAS icing mitigation are evaluated under various icing conditions (i.e., ranged from dry rime icing to wet glaze icing conditions). The findings derived from the detailed icing physics studies can be used to improve current icing accretion models for more accurate prediction of ice formation and accretion process over the surfaces of UAS wings/Propellers as well as to develop effective anti-/de-icing strategies for safer and more efficient UAS operation in cold weather. 